RAIRO Operations Research

RAIRO Oper. Res. 39 (2005) 253-273

DOI: $10.1051 /$ ro:2006005

\title{
A NOTE ON MINTY TYPE VECTOR VARIATIONAL INEQUALITIES
}

\author{
Giovanni P. Crespi $^{1}$, Ivan Ginchev ${ }^{2}$ and Matteo Rocca ${ }^{3}$
}

\begin{abstract}
The existence of solutions to a scalar Minty variational inequality of differential type is usually related to monotonicity property of the primitive function. On the other hand, solutions of the variational inequality are global minimizers for the primitive function. The present paper generalizes these results to vector variational inequalities putting the Increasing Along Rays (IAR) property into the center of the discussion. To achieve that infinite elements in the image space $Y$ are introduced. Under quasiconvexity assumptions we show that solutions of generalized variational inequality and of the primitive optimization problem are equivalent. Finally, we discuss the possibility to generalize the scheme of this paper to get further applications in vector optimization.
\end{abstract}

Keywords. Minty vector variational inequality, existence of solutions, increasing-along-rays property, vector optimization.

Mathematics Subject Classification. 47J20, 49J52, 90C29

\section{INTRODUCTION}

Variational inequalities (for short, VI) provide suitable mathematical models for a range of practical problems, see e.g. [1] or [11]. Vector VI were introduced first in [6] and thereafter studied intensively (for a survey and some recent results we

\footnotetext{
Received November 10, 2004. Accepted January 6, 2006.

${ }^{1}$ Université de la Vallé d'Aoste, Faculty of Economics, 11100 Aosta, Italy;

g.crespi@univda.it

2 Technical University of Varna, Department of Mathematics, 9010 Varna, Bulgaria;

ginchev@ ms3.tu-varna.acad.bg

${ }^{3}$ University of Insubria, Department of Economics, 21100 Varese; Italy;
}

mrocca@eco.uninsubria.it

(c) EDP Sciences 2006 
refer to [7,13]). Two alternative formulations, known as Stampacchia VI [20] and Minty VI [16], are widely studied. In both cases some interest comes from differential VI, where the operator involved defines some directional derivative. Under this setting, Minty VI, compared to Stampacchia VI, characterize more qualified equilibria, that is when a Minty VI admits a solution, then the primitive function has some regularity properties. In [4] we observe that such is the increasing-alongrays (IAR) property. The aim of the present paper is to generalize this result to vector Minty VI.

In [4] we studied the inequality

$$
f_{-}^{\prime}\left(x, x^{*}-x\right) \leq 0, \quad x \in K
$$

where $f: K \rightarrow \boldsymbol{R}$ and $K$ is a subset of the linear space $X$. Here $f_{-}^{\prime}(x, u)$ denotes the lower Dini directional derivative of $f$ in direction $u \in X$ defined for $x \in \operatorname{dom} f$ as an element of $\bar{R}=\boldsymbol{R} \cup\{-\infty\} \cup\{+\infty\}$ by

$$
f_{-}^{\prime}(x, u)=\liminf _{t \rightarrow 0^{+}} \frac{1}{t}(f(x+t u)-f(x)) .
$$

We proved the following properties are related:

(1) $x^{*}$ is a solution of the scalar variational inequality;

(2) $f$ is increasing along rays starting at $x^{*}$;

(3) $x^{*}$ is a global solution of the optimization problem $\min f(x), x \in K$.

In this paper we focus on the IAR property and establishes similar relations for a vector Minty VI. The development of the argument requires, in analogy with [4], to introduce infinite elements in the image space $Y$. The construction proves to be consistent with the VI and applies to the study of both the VI and the related vector optimization problem. In the literature, while infinite elements are widely used in scalar optimization, this is not the case in vector optimization.

As a whole, like in [17], we base our investigation on methods of nonsmooth analysis. By showing that the IAR property is into the nature of the problem, in fact we relate the paper to $[2,15,21,22]$, where VI are investigated in the framework of generalized monotonicity and generalized convexity.

\section{Preliminaries}

In the sequel $X$ denotes a real linear space and $K$ is a subset of $X$. Further $Y$ is a real topological vector space and $C \subset Y$ is a proper closed convex cone with nonempty interior. Proper means $\emptyset \neq C \neq Y$. Occasionally, we deal with the case of $Y$ finite-dimensional, when we do so, we set $Y=\boldsymbol{R}^{m}$ with some positive integer $m$.

For a given function $f: U \rightarrow Y$, where $K \subset U \subset Y$, we consider the vector VI

$$
f^{\prime}\left(x, x^{*}-x\right) \cap(-C) \neq \emptyset, \quad x \in K
$$


where $f^{\prime}(x, u)$ is the Dini (directional) derivative (for short, the adjective directional will be omitted) of $f$ at $x$ in the direction $u$, defined as the set

$$
f^{\prime}(x, u)=\operatorname{Limsup}_{t \rightarrow 0^{+}} \frac{1}{t}(f(x+t u)-f(x))
$$

with the Painlevée-Kuratowski limit. In other words $y \in f^{\prime}(x, u)$ if $y \in Y$ and there exists a sequence $t_{k} \rightarrow 0^{+}$such that

$$
y=\lim _{k} \frac{1}{t_{k}}\left(f\left(x+t_{k} u\right)-f(x)\right) .
$$

The usefulness of the Dini derivative (3) has been proved in [9], where it is applied to derive optimality conditions for $C^{0,1}$ vector minimization problems, i.e. for problems with locally Lipschitz data.

A solution of (2) is any point $x^{*} \in K$ such that $f^{\prime}\left(x, x^{*}-x\right) \cap(-C) \neq \emptyset$ for all $x \in K$. We recall that, for a given set $K \subset X$, the kernel of $K(\operatorname{ker} K)$ is the set of all $x^{*} \in K$, such that $\left[x^{*}, x\right] \subset K$ for all $x \in K$. Obviously ker $K$ is convex. A nonempty set $K$ is star-shaped if $\operatorname{ker} K \neq \emptyset$. It is convenient also to consider the empty set as a star-shaped set. For a survey on star-shaped analysis we refer to [18].

If we look for solutions $x^{*}$ of (2), such that $x^{*} \in \operatorname{ker} K$, then we may assume that $f$ is defined on $K$, since the Dini derivatives in (2) do not depend on the values of $f$ outside $K$.

Together with the vector VI we consider the optimization problem

$$
\min _{C} f(x), \quad x \in K .
$$

Solutions of (4) are intended as global optima. Following [14] we introduce the following type of solutions. A point $x^{*} \in K$ is said to be a $w$-minimizer (weakly efficient point) if $f(K) \cap\left(f\left(x^{*}\right)-\operatorname{int} C\right)=\emptyset$. A point $x^{*} \in K$ is said to be an $e$-minimizer (efficient point) if $f(K) \cap\left(f\left(x^{*}\right)-(C \backslash\{0\})\right)=\emptyset$. A point $x^{*} \in K$ is said to be an $a$-minimizer (absolute or ideal efficient point) if $f(K) \subset f\left(x^{*}\right)+C$.

For scalar functions $f: K \rightarrow \boldsymbol{R}$ we say that $f$ is increasing-along-rays starting at $x^{*} \in \operatorname{ker} K$, we write $f \in I A R\left(K, x^{*}\right)$, when for any $x \in K$ the function $\varphi:[0,1] \rightarrow \boldsymbol{R}, \varphi(t)=f\left((1-t) x^{*}+t x\right)$ is increasing. We say also that $f$ obeys the IAR property.

In [4] we establish under assumption of radially lower semicontinuity of $f$, that $x^{*} \in \operatorname{ker} K$ is a solution of the scalar VI (1) if and only if $f \in I A R\left(K, x^{*}\right)$. The main purpose of the present paper is to generalize results from [4] to a vector VI. For this purpose we have to define first the IAR property for vector-valued functions. In fact we propose two definitions.

Let $x(t)=(1-t) x^{*}+t x$ for given $x^{*}$. We say that $f: K \rightarrow Y$ is increasingalong-rays (minus) starting at $x^{*} \in \operatorname{ker} K$, and write $f \in I A R^{-}\left(K, x^{*}\right)$, if for each $x \in K$ and $0 \leq t_{1}<t_{2} \leq 1$ it holds $f\left(x\left(t_{2}\right)\right) \notin f\left(x\left(t_{1}\right)\right)-\operatorname{int} C$. We say that $f$ is increasing-along-rays (plus) starting at $x^{*} \in \operatorname{ker} K$, and write $f \in I A R^{+}\left(K, x^{*}\right)$, 
if for each $x \in K$ and $0 \leq t_{1}<t_{2} \leq 1$ it holds $f\left(x\left(t_{2}\right)\right) \in f\left(x\left(t_{1}\right)\right)+C$. When $f: K \rightarrow \boldsymbol{R}$ both $\mathrm{IAR}^{-}$and $\mathrm{IAR}^{+}$properties coincide with the IAR property of $f$. Clearly $\mathrm{IAR}^{+}$implies $\mathrm{IAR}^{-}$.

By means of the cone $C$ we can introduce lower semicontinuity of $f: T \rightarrow$ $Y$, where $T$ is a topological space (further we use the abbreviation lsc for lower semicontinuous). We say that $f: T \rightarrow Y$ is lsc at $x^{0}$ if for each $y^{0} \in Y$ such that $f\left(x^{0}\right) \notin y^{0}-C$ there exists a neighborhood $U$ of $x^{0}$ such that $f(U) \cap\left(y^{0}-C\right)=\emptyset$. The function $f: T \rightarrow Y$ is lsc if it is lsc at each point $x^{0} \in T$. To underline the dependence on $C$, we write also $C$-lsc instead of lsc.

Let $f: K \rightarrow Y$ and $x^{*} \in$ ker $K$. We say that $f$ is radially lsc along rays starting at $x^{*}$, and write $f \in R L S C\left(K, x^{*}\right)$, if for all $x \in K$ the function $\varphi:[0,1] \rightarrow Y$, $\varphi(t)=f(x(t))$, is lsc.

\section{The WeIERSTRASS THEOREM FOR LSC VECTOR FUNCTIONS}

Theorem 2 in this section establishes a relation between the existence of solutions of the vector VI (2) and the IAR ${ }^{-}$property. For this purpose we need the following variant of the Weierstrass Theorem for lsc vector functions.

Theorem 1. Let $T$ be a compact topological space, $C \subset Y$ be a closed convex cone with nonempty interior, and $f: T \rightarrow Y$ be a lsc function. Then $f$ possesses an e-minimizer in $T$.

Proof. We base the proof on Theorem 3.3, Chapter 2 in [14], according to which it suffices to show that $f(T)$ is $C$-complete set (Def. 3.2, Chapter 2 in [14]), to prove $f$ possesses an $e$-minimizer in $T$, i.e. $f(T)$ possesses no covering of the form $\left.\left\{\left(y_{\alpha}\right)-C\right)^{c} \mid \alpha \in I\right\}$ with $\left\{y_{\alpha}\right\}$ being a decreasing net in $f(T)$. Suppose to the contrary, that there is a decreasing net $\left\{f\left(x_{\alpha}\right) \mid \alpha \in I\right\}$, for which the family $\left\{V_{\alpha} \mid \alpha \in I\right\}$ with $V_{\alpha}=\left(f\left(x_{\alpha}\right)-C\right)^{c}$ is a covering of $f(T)$. Since $f$ is lsc, the family $\left\{f^{-1}\left(V_{\alpha}\right) \mid \alpha \in I\right\}$ is an open covering of $T$. As $T$ is compact, we may extract a finite subcovering $\left\{f^{-1}\left(V_{\alpha}\right) \mid i=1, \ldots, n\right\}$, whence $\left\{V_{\alpha_{i}} \mid i=1, \ldots, n\right\}$ is a subcovering of $f(T)$. By choosing $\alpha>\alpha_{i}$ for $i=1, \ldots, n$, we deduce that $f\left(x_{\alpha}\right)$ belongs to no $V_{\alpha_{i}}, i=1, \ldots, n$, a contradiction.

Theorem 2. Let $f: K \rightarrow Y, x^{*} \in \operatorname{ker} K$, and $f \in R L S C\left(K, x^{*}\right)$. If $x^{*}$ is a solution of the vector VI (2), then $f \in I A R^{-}\left(K, x^{*}\right)$ and $x^{*}$ is a w-minimizer of problem (4).

Proof. Fix $x \in K$ and denote $x(t)=(1-t) x^{*}+t x, 0 \leq t \leq 1$. Observe that for $0<t \leq 1$ it holds $f^{\prime}\left(x(t), x^{*}-x\right) \cap(-C) \neq \emptyset$. Indeed,

$$
f^{\prime}\left(x(t), x^{*}-x(t)\right)=f^{\prime}\left(x(t), t\left(x^{*}-x\right)\right)=t f^{\prime}\left(x(t), x^{*}-x\right) .
$$

Here we apply positive homogeneity of the Dini derivative, which follows from (3). Since $f^{\prime}\left(x(t), x^{*}-x(t)\right) \cap(-C) \neq \emptyset$, we get $f^{\prime}\left(x(t), x^{*}-x\right) \cap(-C) \neq \emptyset$. 
We prove now that $f \in I A R^{-}\left(K, x^{*}\right)$. Let $0 \leq t_{1}<t_{2} \leq 1$. Define the function $\varphi:\left[t_{1}, t_{2}\right] \rightarrow \boldsymbol{R}$ by

$$
\varphi(t)=f(x(t))-\frac{t_{2}-t}{t_{2}-t_{1}} f\left(x\left(t_{1}\right)\right)-\frac{t-t_{1}}{t_{2}-t_{1}} f\left(x\left(t_{2}\right)\right) .
$$

Since $\varphi$ is lsc, according to Theorem 1 there exists a point $\hat{t} \in\left[t_{1}, t_{2}\right]$ such that $\hat{t}$ is an $e$-minimizer, hence also a $w$-minimizer of $\varphi$. We may assume that $\hat{t} \neq t_{1}$ (hence $\left.0 \leq t_{1}<\hat{t} \leq t_{2} \leq 1\right)$. Indeed, we have $\varphi\left(t_{1}\right)=\varphi\left(t_{2}\right)=0$. Therefore, if $t=t_{1}$ is a $w$-minimizer of $\varphi$, also $\hat{t}=t_{2}$ is a $w$-minimizer of $\varphi$. From the definition of $\hat{t}$ for $0<s<\hat{t}-t_{1}$ we have $\varphi(\hat{t}-s) \notin \varphi(\hat{t})-\operatorname{int} C$, whence $\varphi^{\prime}(\hat{t},-1) \cap(-\operatorname{int} C)=\emptyset$. Put $\hat{x}=x(\hat{t})$. The Dini derivative is

$$
\begin{gathered}
\varphi^{\prime}(\hat{t},-1)=\underset{s \rightarrow 0^{+}}{\operatorname{Limsup}} \frac{1}{s}(\varphi(\hat{t}-s)-\varphi(\hat{t})) \\
=\operatorname{Limsup}_{s \rightarrow 0^{+}} \frac{1}{s}\left(f\left(\hat{x}+s\left(x^{*}-x\right)\right)-f(\hat{x})\right)-\frac{1}{t_{2}-t_{1}} f\left(x\left(t_{1}\right)\right)+\frac{1}{t_{2}-t_{1}} f\left(x\left(t_{2}\right)\right) \\
=f^{\prime}\left(\hat{x}, x^{*}-x\right)-\frac{1}{t_{2}-t_{1}} f\left(x\left(t_{1}\right)\right)+\frac{1}{t_{2}-t_{1}} f\left(x\left(t_{2}\right)\right) \subset(-\operatorname{int} C)^{c} .
\end{gathered}
$$

For $\hat{y} \in f^{\prime}\left(x(\hat{t}), x^{*}-x\right) \cap(-C)$ we have

$$
\begin{gathered}
f\left(x\left(t_{2}\right)\right) \in f\left(x\left(t_{1}\right)\right)-\left(t_{2}-t_{1}\right) \hat{y}+(-\operatorname{int} C)^{c} \\
\subset f\left(x\left(t_{1}\right)\right)+C+(-\operatorname{int} C)^{c} \subset f\left(x\left(t_{1}\right)\right)+(-\operatorname{int} C)^{c} .
\end{gathered}
$$

To see this we apply the inclusion $C+(-\operatorname{int} C)^{c} \subset(-\operatorname{int} C)^{c}$. This holds true since, by contradiction $c^{1}+c^{2}=c^{0} \in-\operatorname{int} C$ for some $c^{1} \in C$ and $c^{2} \in(-\operatorname{int} C)^{c}$ implies $c^{2}=c^{0}-c^{1} \in-\operatorname{int} C-C \subset-\operatorname{int} C$, which is a contradiction. Thus $f\left(x\left(t_{2}\right)\right) \in f\left(x\left(t_{1}\right)\right)+(-\operatorname{int} C)^{c}$, which shows that $f \in I A R^{-}\left(K, x^{*}\right)$. In particular for $t_{1}=0, t_{2}=1$ we obtain $f(x) \notin f\left(x^{*}\right)-\operatorname{int} C$ for all $x \in K$. Therefore $x^{*}$ is a $w$-minimizer for problem (4).

The next corollary is the one-dimensional case of Theorem 2 for the scalar VI

$$
f^{\prime}\left(x, x^{*}-x\right) \cap\left(-\boldsymbol{R}_{+}\right) \neq \emptyset, \quad x \in K .
$$

Let us underline that, while $\mathrm{IAR}^{-}$reduces to IAR, (5) and (1) are not equivalent. Later we discuss this matter in details.

Corollary 1. Let $f: K \rightarrow \boldsymbol{R}, x^{*} \in \operatorname{ker} K$ and $f \in R L S C\left(K, x^{*}\right)$. Suppose that $x^{*}$ is a solution of the scalar VI (5). Then $f \in I A R\left(K, x^{*}\right)$ and $x^{*}$ is a minimizer of the associated optimization problem (4).

The next example shows that without the assumption of lsc type, Theorem 2 does not hold. 
Example 1. Let $X=\boldsymbol{R}, K=[0,1], Y=\boldsymbol{R}, C=\boldsymbol{R}_{+}$and $f: K \rightarrow Y$ be given by

$$
f(x)=\left\{\begin{array}{lll}
x & , & 0 \leq x \leq 1 / 2 \\
x-1 / 2 & , & 1 / 2<x \leq 1
\end{array}\right.
$$

Then $x^{*}=0$ is a solution of the scalar VI (5) but $f \notin I A R\left(K, x^{*}\right)$.

Like in [4], we would expect any definition of IAR property associated with vector functions to be necessary and sufficient condition for $x^{*}$ to solve 2 . Unfortunately this is not the case with $\mathrm{IAR}^{-}$property as Example 2 shows. This leads us to investigate also $\mathrm{IAR}^{+}$property.

Example 2. Let $X=\boldsymbol{R}, K=X, Y=\boldsymbol{R}^{2}, C=\boldsymbol{R}_{+}^{2}$, and $f: K \rightarrow Y$ be given by $f(x)=(x,-x)$. Then for any $x^{*} \in K$ we have $f \in \operatorname{IAR}^{-}\left(K, x^{*}\right)$. At the same time $x^{*}$ is not a solution of the vector VI (2). In fact we have $f^{\prime}\left(x, x^{*}-x\right)=\left(x^{*}-x,-\left(x^{*}-x\right)\right) \notin-C$ for $x \neq x^{*}$.

\section{THE VECTOR VI AND THE $\mathrm{IAR}^{+}$PROPERTY}

In this section we show that Theorem 2 can be strengthened. Namely, we show that the assumptions imply the stronger $\mathrm{IAR}^{+}$property.

Denote by $Y^{\prime}$ the dual space of the topological vector space $Y$ and by

$$
C^{\prime}=\left\{\xi \in Y^{\prime} \mid\langle\xi, y\rangle \geq 0 \text { for all } y \in C\right\}
$$

the positive polar cone of $C$. By assumption $C$ is a proper closed convex cone with nonempty interior. Due to the Separation Theorem for topological vector spaces, see Theorem 9.1 in [19], we have $C=\left\{y \in Y \mid\langle\xi, y\rangle \geq 0\right.$ for all $\left.\xi \in C^{\prime}\right\}$. The following Lemma is straightforward.

Lemma 1. Let $x^{*} \in \operatorname{ker} K$ and $f: K \rightarrow Y$. Then $f \in I A R^{+}\left(K, x^{*}\right)$ if and only if $\langle\xi, f\rangle \in \operatorname{IAR}\left(K, x^{*}\right)$ for all $\xi \in C^{\prime}$.

Further we replace the assumption $f \in R L S C\left(K, x^{*}\right)$ with the stronger $\langle\xi, f\rangle \in$ $R L S C\left(K, x^{*}\right)$ for all $\xi \in C^{\prime}$. Here lower semicontinuity is in the usual sense. In other words $\langle\xi, f\rangle \in R L S C\left(K, x^{*}\right)$ means that $\langle\xi, f\rangle: K \rightarrow \boldsymbol{R}$ is radially lsc along rays starting at $x^{*}$ with regard to the cone $\boldsymbol{R}_{+}$in $\boldsymbol{R}$. The next lemma relates the two notions of lower semicontinuity.

Lemma 2. Let $x^{*} \in \operatorname{ker} K, f: K \rightarrow Y$ and $\langle\xi, f\rangle \in R L S C\left(K, x^{*}\right)$ for all $\xi \in C^{\prime}$. Then $f \in R L S C\left(K, x^{*}\right)$.

Proof. Let $x \in X$ and $y^{*} \in Y$ be such that $f(x) \notin\left(y^{*}-C\right)$. Then $f(x)-y^{*} \notin$ $-C$. Therefore, there exists $\xi \in C^{\prime}$ such that $\left\langle\xi, f(x)-y^{*}\right\rangle>0$ or, equivalently, $\langle\xi, f(x)\rangle>\left\langle\xi, y^{*}\right\rangle$. Since $\langle\xi, f\rangle \in R L S C\left(K, x^{*}\right)$, there exists a neighborhood $U$ of $x$, such that $\langle\xi, f(U)\rangle \cap\left(\left\langle\xi, y^{*}\right\rangle-\operatorname{int} \boldsymbol{R}_{+}\right)=\emptyset$. Now obviously $f(U) \subset\left(y^{*}-\operatorname{int} C\right)^{c}$, which shows that $f \in R L S C\left(K, x^{*}\right)$.

The following example shows Lemma 2 cannot be reverted. 
Example 3. Let $X=\boldsymbol{R}, K=[0,1], Y=\boldsymbol{R}^{2}, C=\boldsymbol{R}_{+}^{2}$ and $f: K \rightarrow Y$ is given by

$$
f(x)= \begin{cases}(1 /(1-x),-1) & , \quad 0 \leq x<1, \\ (0,0) & , \quad x=1 .\end{cases}
$$

Then for $x^{*}=1 f \in R L S C\left(K, x^{*}\right)$ holds, but it is not true that $\langle\xi, f\rangle \in$ $R L S C\left(K, x^{*}\right)$ for all $\xi \in C^{\prime}$. If $\xi=(0,1)$ then $\langle\xi, f\rangle$ fails to be lsc at $x^{*}=1$.

Together with the vector VI (2) we study the scalarized VI

$$
(\langle\xi, f\rangle)^{\prime}\left(x, x^{*}-x\right) \cap\left(-\boldsymbol{R}_{+}\right) \neq \emptyset, \quad x \in K, \quad \text { for all } \xi \in C^{\prime} .
$$

Let us mention that

$$
\left\langle\xi, f^{\prime}\left(x, x^{*}-x\right)\right\rangle \subseteq(\langle\xi, f\rangle)^{\prime}\left(x, x^{*}-x\right)
$$

but, in general, these two sets are different.

Lemma 3. If $x^{*} \in K$ is a solution of the vector VI (2), then $x^{*}$ is also a solution of the scalarized VI (6).

Proof. Let $x^{*}$ be a solution of the vector VI (2) and $x \in K$. Then there exists $y^{*} \in f^{\prime}\left(x, x^{*}-x\right)$ such that $y^{*} \in-C$. The latter means that $\left\langle\xi, y^{*}\right\rangle \leq 0$ for all $\xi \in C^{\prime}$. Since obviously also $\left\langle\xi, y^{*}\right\rangle \in\left\langle\xi, f^{\prime}\left(x, x^{*}-x\right)\right\rangle \subset(\langle\xi, f\rangle)^{\prime}\left(x, x^{*}-x\right)$, we see that $x^{*}$ is a solution also of $(6)$.

The next theorem gives a relation between existence of solutions of the vector VI (2) and the $\mathrm{IAR}^{+}$property.

Theorem 3. Let $f: K \rightarrow Y, x^{*} \in \operatorname{ker} K$, and $\langle\xi, f\rangle \in R L S C\left(K, x^{*}\right)$ for all $\xi \in C^{\prime}$. Suppose that $x^{*}$ is a solution of the vector VI (2). Then $f \in I A R^{+}\left(K, x^{*}\right)$ and $x^{*}$ is an a-minimizer of the associated optimization problem (4).

Proof. According to Lemma 3 the point $x^{*}$ is a solution of the scalarized VI (6). Fix $\xi \in C^{\prime}$. We see that the hypotheses of Corollary 1 are satisfied. The conclusion gives $\langle\xi, f\rangle \in \operatorname{IAR}\left(K, x^{*}\right)$. From Lemma 1 we get $f \in I A R^{+}\left(K, x^{*}\right)$.

The assumptions $\langle\xi, f\rangle \in R L S C\left(K, x^{*}\right)$ for all $\xi \in C^{\prime}$, is stronger than $f \in$ $R L S C\left(K, x^{*}\right)$ in Theorem 2. We leave, as an open question, whether Theorem 3 and the forthcoming results for vector functions remain true under the weaker assumption $f \in R L S C\left(K, x^{*}\right)$.

\section{INFINITE ELEMENTS AND THE EXTENDED VI}

As we did for $\mathrm{IAR}^{-}$property, we try now to work out a reversal of Theorem 3. Some negative answer to the attempt to have a straightforward result are in the next example. 
Example 4. Let $X=\boldsymbol{R}, K=[0,1], Y=\boldsymbol{R}^{2}, C=\boldsymbol{R}_{+}^{2}$. Let $f: K \rightarrow Y$ be defined by $f(x)=(\varphi(x), \varphi(x))$, where $\varphi:[0,1] \rightarrow \boldsymbol{R}$ is any monotonic increasing singular function, for instance let $\varphi$ be the well known Cantor scale function [12]. Recall that $\varphi$ is continuous and defines a measure on $[0,1]$, whose support is a set $\mathcal{C}=\operatorname{supp} \varphi \subset[0,1]$ with Lebesgue measure zero. Then $[0,1] \backslash \mathcal{C}=\bigcup_{i=1}^{\infty} I_{i}$, where $I_{i}$ are mutually disjoint open intervals. Put $x^{*}=0$. For $x \in I_{i}$, for $x$ a right end of the closure of an interval $I_{i}$, and for $x=0$, we have $\varphi^{\prime}\left(x, x^{*}-x\right)=0$ and $f^{\prime}\left(x, x^{*}-x\right)=(0,0)$. For all the other points in $\mathcal{C}$ the Dini derivative $\varphi^{\prime}\left(x, x^{*}-x\right)$ is empty (but the lower Dini derivative is $\left.\varphi_{-}^{\prime}\left(x, x^{*}-x\right)=-\infty\right)$ and $f^{\prime}\left(x, x^{*}-x\right)=\emptyset$. Consequently, $x^{*}$ is not a solution of the vector VI (2), in spite that $f \in I A R^{+}\left(K, x^{*}\right)$.

This situation can be observed also in the scalar case.

Example 5. Let $X=\boldsymbol{R}, K=[0,1], Y=\boldsymbol{R}, C=\boldsymbol{R}_{+}$. Let $f=\varphi$, where $\varphi$ is the singular function from Example 4. Put $x^{*}=0$. Then $f \in \operatorname{IAR}\left(K, x^{*}\right)$. The point $x^{*}$ is a solution of the VI (1), but $x^{*}$ does not solve the scalar VI (5).

In view of Example 5, let us underline that while the scalar VI (1) implies infinities, (5) does not. From this point of view (1) is in some sense an extension of (5) with infinite elements. The usage of infinities is the main difference, which causes the different behavior occurring in Example 5. Actually, in [4] we claimed that $f \in \operatorname{IAR}\left(K, x^{*}\right)$ implies that $x^{*}$ is a solution of (1), the extended VI. In order to observe a similar situation in the vector case, we have first to extend $Y$ with infinite elements, and thereafter to extend the vector VI (2).

We introduce the set of infinite elements $Y_{\infty}=\left\{v_{\infty} \mid v \in Y \backslash\{0\}\right\}$. The element $v_{\infty}$ will be interpreted as infinite element in direction $v$. We accept that $v_{\infty}^{1}=v_{\infty}^{2}$ if and only if $v^{2}=\lambda v^{1}$ for some $\lambda>0$. We put $\tilde{Y}=Y \cup Y_{\infty}$.

A topology on $\tilde{Y}$ can be introduced in terms of local bases of neighborhoods. If $y \in Y$ and $\mathcal{B}(y)$ is a local base of neighborhoods of $y$ in $Y$, we accept that $\mathcal{B}(y)$ is also a local base of neighborhoods of $y$ in $\tilde{Y}$. The family $\mathcal{B}\left(v_{\infty}\right)=\left\{(y+W) \cup W_{\infty} \mid\right.$ $y \in Y, v \in W, W$ open cone in $Y\}$ is a local base of neighborhoods of $v_{\infty}$. Here $W_{\infty}=\left\{w_{\infty} \mid w \in W \backslash\{0\}\right\}$. By saying that $W$ is an open cone in $Y$, we mean that $W$ is an open set in $Y$ such that $\lambda W \subset W$ for all $\lambda>0$.

The extended topological space $\tilde{Y}$ has the following important property.

Proposition 1. When $Y=\boldsymbol{R}^{m}$ the space $\tilde{Y}$ is compact.

We omit the details and give only a sketch of the proof. Consider open covering of $\tilde{Y}$ consisting of sets from the base described. Then there is a finite subfamily of the covering $\left\{\left(y^{i}+W^{i}\right) \cup W_{\infty}^{i} \mid i=1, \ldots, k\right\}$, where $W^{i}$ are open cones, such that $Y_{\infty}$ is a subset of the union of these sets. To see this, it is enough to choose $W^{i}$, $i=1, \ldots, k$, so that their union covers the unit sphere in $Y$. To finish the proof we observe that the set $Y \backslash \cup_{i}\left(y^{i}+W^{i}\right)$ is compact.

Since $\tilde{Y}$ is a topological space, we can apply topological operations on $\tilde{Y}$. Obviously $\operatorname{cl} C=\tilde{C}:=C \cup C_{\infty}$, where $C_{\infty}=\left\{v_{\infty} \mid v \in C \backslash\{0\}\right\}$. We have also 
$\operatorname{int} \tilde{C}=\operatorname{int} C \cup C_{\infty}^{\circ}$, where $C_{\infty}^{\circ}=\left\{v_{\infty} \mid v \in \operatorname{int} C\right\}$. In general topological operations in $Y$ lead to different results than those in $\tilde{Y}$, e.g. in $Y \operatorname{cl} C=C$ holds. To avoid ambiguity, we will highlight when topological operations in $\tilde{Y}$ are applied.

For a function $f: X \rightarrow \tilde{Y}$ the domain $\operatorname{dom} f$ is defined by $\operatorname{dom} f=\{x \in X \mid$ $f(x) \in Y\}$.

For a function $f: X \rightarrow \tilde{Y}$ we extend the Dini derivative $f^{\prime}(x, u)$ for $x \in \operatorname{dom} f$ and $u \in X$ by (3), where the Limsup is calculated in $\tilde{Y}$.

Introducing the Dini derivative in $\tilde{Y}$ we get the opportunity to extend in a natural way the vector VI (2) to

$$
f^{\prime}\left(x, x^{*}-x\right) \cap(-\tilde{C}) \neq \emptyset, \quad x \in K .
$$

Here, as in (2), $f: U \rightarrow Y$, where $K \subset U \subset Y$ and $U$ is a neighborhood of $K$. A solution of (7) is said to be any point $x^{*} \in K$ such that $f^{\prime}\left(x, x^{*}-x\right) \cap(-\tilde{C}) \neq \emptyset$ for all $x \in K$. If we are looking for a solution $x^{*} \in \operatorname{ker} K$ of (2), then we may assume that $f$ is defined on $K$.

Remark 1. Each solution of (2) is a solution of (7), but Example 4 shows that the reverse is not true. Indeed if we calculate in $\tilde{Y}$ the Dini derivatives $f^{\prime}\left(x, x^{*}-x\right)$, we have $f^{\prime}\left(x, x^{*}-x\right)=(0,0) \in-\tilde{C}$ for $x \in I_{i, j}$, for $x$ a right end of the closure of an interval $I_{i, j}$, or for $x=0$. For any other point in $C$ it holds $f^{\prime}\left(x, x^{*}-x\right)=$ $-(1,1)_{\infty} \in-\tilde{C}$. Therefore $f \in I A R^{+}\left(K, x^{*}\right)$ and $x^{*}$ solves the extended vector VI (7). Hence (7) is a generalization of (2). When $Y=\boldsymbol{R}$ the sets of the solutions of (7) and (1) coincide, while, in general, this is not the case if we compare (2) and (1). This has been the main motivation to extend $Y$ to $\tilde{Y}$ and the vector VI (2) to (7).

Theorem 4. Let $Y=\boldsymbol{R}^{m}, f: K \rightarrow Y$, and $x^{*} \in \operatorname{ker} K$. Suppose that $f \in$ $I A R^{+}\left(K, x^{*}\right)$. Then $x^{*}$ is a solution of the extended vector VI (7).

Proof. Fix $x \in K$ and let $x(t)=(1-t) x^{*}+t x, 0 \leq t \leq 1$. For $0<t<1$ we have

$$
\frac{1}{t}\left(f\left(x+t\left(x^{*}-x\right)\right)-f(x)\right)=\frac{1}{t}(f(x(1-t))-f(x(1))) \in-C .
$$

Since $\operatorname{cl} C=\tilde{C}$ (the closure in $\tilde{Y}$ ), from (3) we get $f^{\prime}\left(x, x^{*}-x\right) \subset-\tilde{C}$. To assure that $x^{*}$ is a solution of the extended VI (7) it remains to show that $f^{\prime}\left(x, x^{*}-x\right) \neq$ $\emptyset$. This is however a consequence of the compactness of $\tilde{Y}$ (in a compact topological space every sequence has a cluster point).

Let us underline that Theorem 4 does not require lsc type assumption, as it did in Theorem 3.

Since the conclusion of Theorem 4 concerns the extended vector VI (7), we now study Theorem 3 with this formulation of the vector VI.

Remark 2. The class of functions $f$, for which the extended vector VI (7) has a solution is obviously larger than the class of functions for which the vector VI (2) has a solution. 
Theorem 5. Let $f: K \rightarrow Y, x^{*} \in \operatorname{ker} K$ and $\langle\xi, f\rangle \in R L S C\left(K, x^{*}\right)$ for all $\xi \in C^{\prime}$. Suppose that $x^{*}$ is a solution of the extended vector VI (7) such that $x^{*}$ satisfies $f^{\prime}\left(x, x^{*}-x\right) \cap(-\operatorname{int} \tilde{C}) \neq \emptyset$ (the interior is taken in $\left.\tilde{Y}\right)$ at the points where $f^{\prime}\left(x, x^{*}-x\right) \cap(-C)=\emptyset$. Then $f \in I A R^{+}\left(K, x^{*}\right)$ and $x^{*}$ is an a-minimizer of the associated optimization problem (4).

Proof. Fix $x \in K$ and denote $x(t)=(1-t) x^{*}+t x, 0 \leq t \leq 1$. The positive homogeneity of the Dini derivative (in $\tilde{Y}$ ) gives $f^{\prime}\left(x(t), x^{*}-x(t)\right)=t f^{\prime}(x(t)$, $\left.x^{*}-x\right)$. Since $x^{*}$ satisfies the hypotheses, we get that for $0<t \leq 1 f^{\prime}(x(t)$, $\left.x^{*}-x\right) \cap(-\operatorname{int} \tilde{C}) \neq \emptyset$ holds at the points where $f^{\prime}\left(x(t), x^{*}-x\right) \cap C=\emptyset$.

Fix $\xi \in C^{\prime}$ and let $0 \leq t_{1}<t_{2} \leq 1$. We define the function $\varphi:\left[t_{1}, t_{2}\right] \rightarrow \boldsymbol{R}$ by

$$
\varphi(t)=\langle\xi, f(x(t))\rangle-\frac{t_{2}-t}{t_{2}-t_{1}}\left\langle\xi, f\left(x\left(t_{1}\right)\right)\right\rangle-\frac{t-t_{1}}{t_{2}-t_{1}}\left\langle\xi, f\left(x\left(t_{2}\right)\right)\right\rangle .
$$

Since $\varphi$ is lsc, there exists a point $\hat{t} \in\left[t_{1}, t_{2}\right]$ such that $\varphi(\hat{t})=\min \left\{\varphi(t) \mid t_{1} \leq t \leq\right.$ $\left.t_{2}\right\}$. We may assume that $0 \leq t_{1}<\hat{t} \leq t_{2} \leq 1$ (if $\varphi$ attains minimum at $t_{1}$, due to $\varphi\left(t_{1}\right)=\varphi\left(t_{2}\right)$ it attains minimum also at $\left.t_{2}\right)$. Put $\hat{x}=x(\hat{t})$ and

$$
\hat{y}=\lim _{k} \frac{1}{s_{k}}\left(f\left(\hat{x}+s_{k}\left(x^{*}-x\right)\right)-f(\hat{x})\right) \in f^{\prime}\left(\hat{x}, x^{*}-x\right),
$$

where $s_{k} \rightarrow 0^{+}$. We may assume that $0<s_{k}<\hat{t}-t_{1}$ for all $k$. The minimal property of $\hat{t}$ gives $\varphi\left(\hat{t}-s_{k}\right) \geq \varphi(\hat{t})$, which can be written in the form

$$
\left\langle\xi, \frac{1}{s_{k}}\left(f\left(\hat{x}+s_{k}\left(x^{*}-x\right)\right)-f(\hat{x})\right)\right\rangle+\left\langle\xi, \frac{1}{t_{2}-t_{1}}\left(f\left(x\left(t_{2}\right)\right)-f\left(x\left(t_{1}\right)\right)\right)\right\rangle \geq 0 .
$$

As we have seen above $\hat{y} \in f^{\prime}\left(\hat{x}, x^{*}-x\right)$ can be chosen in such a way that the following alternative has place:

$\mathbf{1}^{0} . \hat{y} \in-C$,

$\mathbf{2}^{0} \cdot \hat{y}=\hat{v}_{\infty} \in-\operatorname{int} \tilde{C}$.

Assume that $1^{0}$ holds. Then passing to a limit in (8) and applying $\langle\xi, \hat{y}\rangle \leq 0$ we get $\left\langle\xi, f\left(x\left(t_{2}\right)\right)-f\left(x\left(t_{1}\right)\right)\right\rangle \geq 0$.

Let assume now that $2^{0}$ holds. Then there exists an open cone $W \subset C$, such that $\hat{v} \in-W \subset-C$. From the definition of convergence in $\tilde{Y}$, for sufficiently large $k$, we have $\left(1 / s_{k}\right)\left(f\left(\hat{x}+s_{k}\left(x^{*}-x\right)\right)-f(\hat{x})\right) \in-W \subset-C$. Therefore

$$
\left\langle\xi, f\left(x\left(t_{2}\right)\right)-f\left(x\left(t_{1}\right)\right)\right\rangle \geq-\left(t_{2}-t_{1}\right)\left\langle\xi, \frac{1}{s_{k}}\left(f\left(\hat{x}+s_{k}\left(x^{*}-x\right)\right)-f(\hat{x})\right)\right\rangle \geq 0 .
$$

Thus, we have shown that $\left\langle\xi, f\left(x\left(t_{2}\right)\right)-f\left(x\left(t_{1}\right)\right)\right\rangle \geq 0$ for all $\xi \in C^{\prime}$. This implies that $f\left(x\left(t_{2}\right)\right)-f\left(x\left(t_{1}\right)\right) \in C$ and therefore $f \in I A R^{+}\left(K, x^{*}\right)$. Putting $t_{1}=0$ and $t_{2}=1$ we get $f(x)-f\left(x^{*}\right) \in C$ for arbitrary $x \in K$. Therefore $x^{*}$ is an $a$-minimizer of $f$ on $K$. 
When $Y=\boldsymbol{R}$ and $C=\boldsymbol{R}_{+}$, the extended space is $\tilde{Y}=\overline{\boldsymbol{R}}=[-\infty,+\infty]$ and $\tilde{C}=\overline{\boldsymbol{R}}_{+}=[0,+\infty]$. In this case the extended VI (7)

$$
f^{\prime}\left(x, x^{*}-x\right) \cap\left(-\overline{\boldsymbol{R}}_{+}\right) \neq \emptyset, \quad x \in K
$$

is equivalent to (1). Therefore also (1) can be regarded as the extension of the scalar VI (5), although we shall consider (9) as the most appropriate formulation. In this setting $\overline{\boldsymbol{R}}_{+}=\operatorname{int} \overline{\boldsymbol{R}}_{+} \cup\{0\}$, which simplifies the formulation of Theorem 5 . As a corollary, we get the following, which is exactly the result established in [4].

Corollary 2. Let $f: K \rightarrow \boldsymbol{R}, x^{*} \in \operatorname{ker} K$, and $f \in R L S C\left(K, x^{*}\right)$. Suppose that $x^{*}$ is a solution of the scalar VI (1). Then $f \in I A R\left(K, x^{*}\right)$ and $x^{*}$ is a global solution of the optimization problem $\min f(x), x \in K$.

In Theorem 4 we get, as a conclusion, that the extended vector VI (7) has a solution $x^{*}$. Therefore we would expect a reversal quoting " $x^{*}$ is a solution of (7)" among the assumptions. Instead Theorem 5 involves the VI

$$
f^{\prime}\left(x, x^{*}-x\right) \cap(-\operatorname{int} \tilde{C}) \neq \emptyset .
$$

The next example shows that in general in Theorem 5 the assumptions concerning the infinite elements in the derivative $f^{\prime}\left(x, x^{*}-x\right)$ cannot be simplified.

Example 6. Let $X=\boldsymbol{R}, K=[0,1], Y=\boldsymbol{R}^{2}, C=\left\{\left(y_{1}, y_{2}\right) \in Y \mid y_{2} \geq 0\right\}$. Let $f: K \rightarrow Y$ be defined by

$$
f(x)= \begin{cases}(x /(1-x), x) & , \quad 0 \leq x<1 \\ (0,0) & , \quad x=1\end{cases}
$$

Let $x^{*}=0$. Then $x^{*} \in \operatorname{ker} K,\langle\xi, f\rangle \in R L S C\left(K, x^{*}\right)$ for all $\xi \in C^{\prime}$, and $x^{*}$ is a solution of the extended vector VI (7). In fact

$$
f^{\prime}\left(x, x^{*}-x\right)= \begin{cases}-\left(x /(1-x)^{2}, x\right) & , \quad 0 \leq x<1 \\ (1,0)_{\infty} & , \quad x=1\end{cases}
$$

At the same time $f \notin I A R^{+}\left(K, x^{*}\right)$.

Recall that the general assumption for $C$ is that $C$ is a proper closed convex cone with nonempty interior. We leave as an open question, whether an example like Example 6 can be constructed with $C$ pointed, i.e. $C \cap(-C)=\{0\}$.

\section{The OPtimization PROBLEM With INFINite ELEMENTS}

The attempt to revert Theorem 3 motivated the definition of $\tilde{Y}$. A topology on $\tilde{Y}$ was needed to consider convergence in $\tilde{Y}$.

When $Y=\boldsymbol{R}, \tilde{Y}=\overline{\boldsymbol{R}}$, a classical tool for nonsmooth analysis is to replace problem

$$
\min f(x), \quad x \in K,
$$


by the equivalent (unconstrained)

$$
\min \tilde{f}(x), \quad x \in X,
$$

where $\tilde{f}: X \rightarrow \overline{\boldsymbol{R}}$ is the extension of $f$ by $\tilde{f}(x)=+\infty$ when $x \in X \backslash K$. In this section we discuss the possibility for a similar transformation of the vector optimization problem (4) with the help of infinite elements of $\tilde{Y}$.

Recall that $C$ is a proper closed convex cone with nonempty interior. Proper means that $C \neq Y$ and implies int $C \cap(-\operatorname{int} C)=\emptyset$. Therefore, we can fix $v^{0} \in \operatorname{int} C$ and we have $-v^{0} \notin \operatorname{int} C$. The elements $v^{0}$ and $-v^{0}$ define the infinite elements $v_{\infty}^{0}$ and $-v_{\infty}^{0}:=\left(-v^{0}\right)_{\infty}$. We will set $\tilde{Y}_{0}=Y \cup\left\{-v_{\infty}^{0}\right\} \cup\left\{v_{\infty}^{0}\right\}$ and $\tilde{C}_{0}=C \cup\left\{v_{\infty}^{0}\right\}$.

We relate to vector optimization problem (4) the equivalent optimization problem

$$
\min _{C} \tilde{f}(x), \quad x \in X,
$$

where $\tilde{f}: X \rightarrow \tilde{Y}_{0}$ is defined by

$$
\tilde{f}(x)= \begin{cases}f(x), & x \in K \\ v_{\infty}^{0}, & x \in X \backslash K .\end{cases}
$$

For problem (10) with arbitrary function $\tilde{f}: X \rightarrow \tilde{Y}_{0}$ we define the solutions $x^{*} \in \operatorname{dom} \tilde{f}$ formally in the same way as those for the finite problem (4). The point $x^{*} \in \operatorname{dom} \tilde{f}$ is a $w$-minimizer if $f(X) \cap\left(f\left(x^{*}\right)-\operatorname{int}_{0} \tilde{C}^{0}\right)=\emptyset$, it is an $e$ minimizer if $f(X) \cap\left(f\left(x^{*}\right)-\left(\tilde{C}^{0} \backslash\{0\}\right)\right)=\emptyset$. We say that the point $x^{*} \in \operatorname{dom} \tilde{f}$ is an $a$-minimizer if $f(X) \subset f\left(x^{*}\right)+\tilde{C}^{0}$. In order to give sense to these notions we introduce the following definitions. We set int $\tilde{C}^{0}=\operatorname{int} C \cup\left\{v_{\infty}^{0}\right\}$. The subscript in int $_{0}$ is to remind that this operation should not be confused with the interior in $\tilde{Y}$, which is in fact int $\tilde{C}^{0}=\operatorname{int} C$. Furthermore for $y \in Y$ we put

$$
\begin{aligned}
y-\operatorname{int}_{0} \tilde{C}^{0} & =(y-\operatorname{int} C) \cup\left\{-v_{\infty}^{0}\right\}, \\
y-\left(\tilde{C}^{0} \backslash\{0\}\right) & =\left(y-(C \backslash\{0\}) \cup\left\{-v_{\infty}^{0}\right\},\right. \\
y+\tilde{C}^{0} & =(y+C) \cup\left\{v_{\infty}^{0}\right\} .
\end{aligned}
$$

Now it is clear that for the optimization problem (10) with $\tilde{f}$ defined by (11) $\operatorname{dom} \tilde{f}=K$ and $x^{*} \in \operatorname{dom} \tilde{f}$ is a $w$-minimizer, $e$-minimizer or $a$-minimizer for problem (10) if and only if $x^{*}$ verifies the same statement for problem (4).

Remark 3. The two-point extension of $Y$ to $\tilde{Y}_{0}$ is in fact identical to the twopoint extension $Y_{C}=Y \cup\left\{+\infty_{C}\right\} \cup\left\{-\infty_{C}\right\}$ of $Y$ suggested in [8]. Though in [8] $+\infty_{C}$ and $-\infty_{C}$ are abstract elements, in fact they can be identified with $v_{\infty}^{0}$ and $(-v)_{\infty}^{0}$ from the above definitions.

If we extend the algebraic operations and the order relations defined by $C$ from $Y$ to $\tilde{Y}$, the definitions we used can be derived from these extensions. Besides, we gain the possibility to treat more general optimization problems (10) with 
functions $\tilde{f}: X \rightarrow \tilde{Y}$. However we will refrain from discussing this extension in the present paper.

In the previous sections we developed the idea that $\mathrm{IAR}^{+}$property may be more fitting with vector Minty VI than $\mathrm{IAR}^{-}$is. However the setting used in Theorems 3 and 4 refrains us from studying solutions outside ker $K$. We try to consider this problem with the aid of $\tilde{f}$.

First we generalize the $\mathrm{IAR}^{-}$and $\mathrm{IAR}^{+}$properties for the function (11). Let $x^{*} \in \operatorname{dom} \tilde{f}$. We write $\tilde{f} \in I_{A}-\left(X, x^{*}\right)$ or $\tilde{f} \in I A R^{+}\left(X, x^{*}\right)$ if for each $x \in$ $\operatorname{dom} \tilde{f}$ it holds $\left[x^{*}, x\right] \subset \operatorname{dom} \tilde{f}$ and, respectively, $\varphi \in I_{A R}^{-}([0,1], 0)$ or $\varphi \in$ $I A R^{+}([0,1], 0)$. Where $\varphi:[0,1] \rightarrow Y, \varphi(t)=f(x(t))$, where $x(t)=(1-t) x^{*}+t x$.

The next example underlines that the property $\tilde{f} \in I A R^{+}\left(X, x^{*}\right)$ has deeper implications than just to say that $\tilde{f}$ increases along the segments starting at $x^{*}$ and contained in $\operatorname{dom} \tilde{f}$.

Example 7. Let $X=\boldsymbol{R}^{2}, K=[(0,0),(1,0)] \cup[(0,0),(0,1)], Y=\boldsymbol{R}, C=\boldsymbol{R}_{+}$. Consider the function (11), where $f: K \rightarrow Y$ is defined by

$$
f\left(x_{1}, x_{2}\right)=\left\{\begin{array}{lc}
0, & x_{2}=0,0 \leq x_{1} \leq 1 / 2 \text { or } \\
& x_{1}=0,0 \leq x_{2} \leq 1 / 2 \\
-2 x_{1}+1, & x_{2}=0,1 / 2 \leq x_{1} \leq 1 \\
-2 x_{2}+1, & x_{1}=0,1 / 2 \leq x_{2} \leq 1
\end{array}\right.
$$

Then $\tilde{f}$ increases along each segment in $\operatorname{dom} \tilde{f}$ starting at $(0,1)$ or $(1,0)$. At the same time $\tilde{f} \notin I A R^{+}\left(X, x^{*}\right)$ for arbitrary $x^{*} \in \operatorname{ker} K$.

For a function $\tilde{f}: X \rightarrow Y \cup\left\{v_{\infty}^{0}\right\}$ we denote by $A(\tilde{f})$ the set of all $a$-minimizers $x^{*} \in \operatorname{dom} \tilde{f}$ and by $S(\tilde{f})$ the set of all $x^{*} \in \operatorname{dom} \tilde{f}$ such that $\tilde{f} \in \operatorname{IAR}^{+}\left(X, x^{*}\right)$. We show that $S(\tilde{f})$ has a simple structure.

Theorem 6. Let $\tilde{f}: X \rightarrow Y \cup\left\{v_{\infty}^{0}\right\}$ and $K=\operatorname{dom} \tilde{f}$. Then it holds $S(\tilde{f}) \subset$ $\operatorname{ker} K \cap A(\tilde{f})$ and the set $S(\tilde{f})$ is convex. If the cone $C$ is pointed, then $f$ takes the same value for all $x \in S(\tilde{f})$.

Proof. Let $x^{*} \in S(\tilde{f})$, that is $\tilde{f} \in I A R^{+}\left(X, x^{*}\right)$. By the definition of $\mathrm{IAR}^{+}$ property, if $x \in K$ then $\left[x^{*}, x\right] \subset K$. That is $x^{*} \subset \operatorname{ker} K$. Further $\varphi(1) \subset \varphi(0)+C$, where $\varphi:[0,1] \rightarrow Y, \varphi(t)=f(x(t))$ with $x(t)=(1-t) x^{*}+t x$. This inclusion can be written as $f(x) \in f\left(x^{*}\right)+C$ for arbitrary $x \in K$. Therefore $x^{*}$ is an $a$-minimizer of problem (10).

Now we prove that the set $S(\tilde{f})$ is convex. Let $x^{0}, x^{1} \in S(\tilde{f})$ and let $\bar{x}=$ $(1-\bar{t}) x^{0}+\bar{t} x^{1}$ with $0<\bar{t}<1$. For $0 \leq t \leq 1$ consider the functions

$$
\begin{gathered}
\bar{x}(t)=(1-t) \bar{x}+t x=(1-t)(1-\bar{t}) x^{0}+(1-t) \bar{t} x^{1}+t x, \\
x^{0}(t)=(1-t) x^{0}+t x, \quad x^{1}(t)=(1-t) x^{1}+t x .
\end{gathered}
$$

It is easy to derive the identities

$$
\bar{x}(t)=\left(1-\alpha_{0}\right) x^{0}+\alpha_{0} x^{1}\left(\beta_{0}\right),
$$


where

$$
\alpha_{0}=t+\bar{t}-t \bar{t}=t+(1-t) \bar{t}, \quad \beta_{0}=\frac{t}{\alpha_{0}}=\frac{t}{t+(1-t) \bar{t}}
$$

and

where

$$
\bar{x}(t)=\left(1-\alpha_{1}\right) x^{1}+\alpha_{1} x^{0}\left(\beta_{1}\right)
$$

$$
\alpha_{0}=1-\bar{t}+t \bar{t}=(1-\bar{t}) t+t \bar{t}, \quad \beta_{1}=\frac{t}{\alpha_{1}}=\frac{t}{1-\bar{t}+t \bar{t}} .
$$

In particular the first identity gives $\bar{x}(t) \in K$ on the base of the following reasoning. Since $x \in K$ and $\tilde{f} \in I A R^{+}\left(X, x^{1}\right)$, we have $x^{1}\left(\beta_{0}\right) \in K$. Since $x^{1}\left(\beta_{0}\right) \in K$ and $\tilde{f} \in I A R^{+}\left(X, x^{0}\right)$, we have $\bar{x}(t) \in K$.

Now we prove that $\tilde{f} \in I A R^{+}(X, \bar{x})$. Fix $0 \leq t_{0}<t_{1} \leq 1$. We must show that $\tilde{f}\left(\bar{x}\left(t_{2}\right)\right) \in \tilde{f}\left(\bar{x}\left(t_{1}\right)\right)+C$. It is easy to check the identity

$$
\left(1-\gamma_{0}\right) x^{0}+\gamma_{0} x^{1}\left(\beta_{0}\right)=\left(1-\gamma_{1}\right) x^{1}+\gamma_{1} x^{0}\left(\beta_{1}\right)
$$

where

We apply this identity for

$$
\gamma_{0}=\frac{\beta_{1}}{\beta_{0}+\beta_{1}-\beta_{0} \beta_{1}}, \quad \gamma_{1}=\frac{\beta_{0}}{\beta_{0}+\beta_{1}-\beta_{0} \beta_{1}} .
$$

$$
\begin{aligned}
& \beta_{0}=t_{0} / \alpha_{0}, \quad \alpha_{0}=t_{0}+\left(1-t_{0}\right) \bar{t}, \\
& \beta_{1}=t_{1} / \alpha_{1}, \quad \alpha_{1}=(1-\bar{t})+t_{1} \bar{t} .
\end{aligned}
$$

In fact $\alpha_{0}, \beta_{0}$ are calculated from (12) for $t=t_{0}$ and $\alpha_{1}, \beta_{1}$ are calculated from (13) for $t=t_{1}$.

We get easily

$$
\begin{aligned}
& \gamma_{0}-\alpha_{0}=\alpha_{0} \frac{\left(t_{1}-t_{0}\right)(1-\bar{t})}{t_{0}+\left(t_{1}-t_{0}\right) \bar{t}}>0, \\
& \alpha_{1}-\gamma_{1}=\alpha_{1} \frac{\left(t_{1}-t_{0}\right) \bar{t}}{t_{0}+\left(t_{1}-t_{0}\right) \bar{t}}>0 .
\end{aligned}
$$

Now it remains to apply the $\mathrm{IAR}^{+}$property. From $\tilde{f} \in I A R^{+}\left(X, x^{0}\right)$ and $\tilde{f} \in$ $I_{A} R^{+}\left(X, x^{1}\right)$ we get respectively

$$
\begin{aligned}
& \tilde{f}\left(\left(1-\gamma_{0}\right) x^{0}+\gamma_{0} x^{1}\left(\beta_{0}\right)\right)-\tilde{f}\left(\bar{x}\left(t_{0}\right)\right) \in C, \\
& \tilde{f}\left(\bar{x}\left(t_{1}\right)\right)-\tilde{f}\left(\left(1-\gamma_{1}\right) x^{1}+\gamma_{1} x^{0}\left(\beta_{1}\right)\right) \in C .
\end{aligned}
$$

Adding the two inclusions we obtain

$$
\tilde{f}\left(\bar{x}\left(t_{1}\right)\right)-\tilde{f}\left(\bar{x}\left(t_{0}\right)\right) \in C+C=C,
$$

which shows that $\tilde{f} \in I A R^{+}(X, \bar{x})$. This proves that the set $S(\tilde{f})$ is convex. 
Finally, let $C$ be pointed and $x^{0}, x^{1} \in S(\tilde{f})$. From $\tilde{f} \in I A R^{+}\left(X, x^{0}\right)$ and $\tilde{f} \in \operatorname{IAR}^{+}\left(X, x^{1}\right)$ we get respectively $\tilde{f}\left(x^{1}\right)-\tilde{f}\left(x^{0}\right) \in C$ and $\tilde{f}\left(x^{0}\right)-\tilde{f}\left(x^{1}\right) \in C$. Therefore

whence $\tilde{f}\left(x^{1}\right)=\tilde{f}\left(x^{0}\right)$.

$$
\tilde{f}\left(x^{1}\right)-\tilde{f}\left(x^{0}\right) \in C \cap(-C)=\{0\},
$$

The next example shows that a VI can have a solution outside ker $K$.

Example 8. Let $X=\boldsymbol{R}^{2}, K=[(0,0),(1,0)] \cup[(0,0),(0,1)], U=\boldsymbol{R}^{2} \backslash\{(t, t) \mid t \geq$ $1 / 3\}, Y=\boldsymbol{R}, C=\boldsymbol{R}_{+}$. Consider the function $f: U \rightarrow \boldsymbol{R}$ defined by

$$
f\left(x_{1}, x_{2}\right)= \begin{cases}0 & , \quad x_{1}+2 x_{2} \leq 1,2 x_{1}+x_{2} \leq 1 \\ -2 a+1 & , \quad x_{1}-x_{2}<0, a x_{1}+x_{2}=a, a \geq 1 / 2, \\ -2 a+1 & , \quad x_{1}-x_{2}>0, x_{1}+a x_{2}=a, a \geq 1 / 2 .\end{cases}
$$

Then ker $K=\{(0,0)\}$ and both $(1,0)$ and $(0,1)$ solve the VI (2).

Usually one expects that in the extended VI (7) the function $f: K \rightarrow Y$ is defined only on $K$. When $x^{*} \notin$ ker $K$ values of $f$ outside $K$ should be given in order to calculate the derivatives in outer directions. Example 8 makes clear that the given values influence the solution. The "greater" the given value, the "smaller" is its influence.

To eliminate that influence, it seems natural to extend $f$ as $v_{\infty}^{0}$ outside $K$. Furthermore, as in the optimization problem, we generalize the VI to the whole space $X$. With a similar approach in [4], we obtained a (scalar)generalized VI, which preserves the solutions in $\operatorname{ker} K$ and has no solutions outside it. Let us underline, for instance, that the function in Example 8 is an extension of that in Example 7. If we would extend the same function with value $+\infty$ outside $K$ the generalized VI will have no solutions.

Now we try to describe a similar approach to vector VI. Let us start from the extended VI (7) with $f: K \rightarrow Y$. From the extended function $\tilde{f}: X \rightarrow \tilde{Y}_{0}$ in (11) we get the generalized VI

$$
\tilde{f}^{\prime}\left(x, x^{*}-x\right) \cap(-\tilde{C}) \neq \emptyset, \quad x \in X,
$$

and look for solutions $x^{*} \in \operatorname{dom} \tilde{f}=K$. In (14) we extend the Dini derivative to the function (11), $\tilde{f}^{\prime}(x, u)$. We define again $f^{\prime}(x, u)$ by $(3)$, where the convergence is in $\tilde{Y}$. For any operation with infinities which may occur, we set for $\lambda>0$, $\lambda v_{\infty}^{0}=v_{\infty}^{0}$ and $\lambda\left(-v_{\infty}^{0}\right)=-v_{\infty}^{0}$. Further for $y \in Y$ we put $v_{\infty}^{0}-y=v_{\infty}^{0}$, $y-v_{\infty}^{0}=-v_{\infty}^{0}, v_{\infty}^{0}-v_{\infty}^{0}=\tilde{Y}_{0}$.

A complete extension of the algebraic operations from $Y$ to $\tilde{Y}$ is beyond the task of this paper. In order to define the Dini derivative of (11) the above definitions are enough. Only the last equality is a bit controversial (related to $\boldsymbol{R}$ this means to accept $(+\infty)-(+\infty)=\overline{\boldsymbol{R}}$, while people usually prefer to give no sense to that difference), but anyway it is not contradictory and it is convenient for our purpose. 
In fact, if $\tilde{f}(x)=v_{\infty}^{0}$ and if $\tilde{f}\left(x+t_{k}\left(x^{*}-x\right)\right)=v_{\infty}^{0}$ for a sequence $t_{k} \rightarrow 0^{+}$, we get $\tilde{f}\left(x, x^{*}-x\right)=\tilde{Y}_{0}$. Therefore, at such a point $x$, condition (14) is satisfied.

Next we relate vector VI (7) to $\mathrm{IAR}^{+}$property of $\tilde{f}$. The result, in some sense, is a repetition of Theorem 4 . The proof follows along the same lines and it is omitted.

Theorem 7. Let $Y=\boldsymbol{R}^{m}$ and let $\tilde{f}: X \rightarrow \tilde{Y}_{0}$ be given by (11). Suppose that $\tilde{f} \in I_{A} R^{+}\left(X, x^{*}\right)$ for some $x^{*} \in K:=\operatorname{dom} \tilde{f}$. Then $x^{*}$ is a solution of the generalized vector $V I(14)$.

Since $\tilde{f}: X \rightarrow \tilde{Y}_{0} \subset \tilde{Y}$ and $\tilde{Y}$ is a topological space, we can define the RLSC property of $\tilde{f}$ as in Section 1. Namely, $\tilde{f} \in R L S C\left(X, x^{*}\right)$ if for all $x \in K$ the function $\varphi:[0,1] \rightarrow \tilde{Y}, \varphi(t)=f(x(t))$, is lsc, where $x(t)=(1-t) x^{*}+t x$. In a similar way we understand the notation $\langle\xi, \tilde{f}\rangle \in R L S C\left(X, x^{*}\right)$.

Now we show that Theorem 5 can be stated for the generalized vector VI (14).

Theorem 8. Let $\tilde{f}: X \rightarrow \tilde{Y}_{0}$ be given by (11), $x^{*} \in K=\operatorname{dom} \tilde{f}$, and $\langle\xi, \tilde{f}\rangle \in$ $R L S C\left(X, x^{*}\right)$ for all $\xi \in C^{\prime}$. Suppose that $x^{*}$ is a solution of the generalized $V I$ (14) such that $x^{*}$ satisfies $\tilde{f}^{\prime}\left(x, x^{*}-x\right) \cap(-\operatorname{int} \tilde{C}) \neq \emptyset$ (the interior is taken in $\tilde{Y})$ at the points $x \in \operatorname{dom} \tilde{f}$ where $\tilde{f}^{\prime}\left(x, x^{*}-x\right) \cap(-C)=\emptyset$. Then $\tilde{f} \in$ $I_{A} R^{+}\left(X, x^{*}\right)$. Further $x^{*} \in \operatorname{ker} K$, and $x^{*}$ is an a-minimizer of the associated optimization problem (10).

Proof. Fix $x \in K$ and put $x(t)=(1-t) x^{*}+t x$. Let $0 \leq t_{1}<t_{2} \leq 1$. We must show that both $\tilde{f}\left(x\left(t_{1}\right)\right)$ and $\tilde{f}\left(x\left(t_{2}\right)\right)$ belong to $Y$, i.e. they are finite, and $\tilde{f}\left(x\left(t_{2}\right)\right) \subset \tilde{f}\left(x\left(t_{1}\right)\right)+C$.

A. First let us show that if both $\tilde{f}\left(x\left(t_{1}\right)\right)$ and $\tilde{f}\left(x\left(t_{2}\right)\right)$ belong to $Y$, then $\tilde{f}\left(x\left(t_{2}\right)\right) \subset \tilde{f}\left(x\left(t_{1}\right)\right)+C$. In fact, this statement is exactly what has been proved in Theorem 5. Following again the proof of Theorem 5, we face the following situation. While in Theorem 5 we have $\varphi(t) \in \boldsymbol{R}$ for $t_{1}<t<t_{2}$, where $\varphi$ is the defined there function, now we have $\varphi(t) \in \boldsymbol{R} \cup\{+\infty\}$. This difference however does not spoil the proof.

B. By contradiction, assume now that $\tilde{f}\left(x\left(t_{1}\right)\right)=v_{\infty}^{0}, \tilde{f}\left(x\left(t_{2}\right)\right) \in Y$.

From $v^{0} \in \operatorname{int} C$ there exists $\lambda_{0}>0$ such that $v^{0}-\left(1 / \lambda_{0}\right) \tilde{f}\left(x\left(t_{2}\right)\right) \in \operatorname{int} C$, or equivalently $\lambda_{0} v^{0} \in \tilde{f}\left(x\left(t_{2}\right)\right)+\operatorname{int} C$.

Fix $\xi \in C^{\prime}$ and define the function $\varphi:\left[t_{1}, t_{2}\right] \rightarrow \boldsymbol{R} \cup\{+\infty\}$ by

$$
\varphi(t)=\langle\xi, \tilde{f}(x(t))\rangle-\lambda_{0} \frac{t_{2}-t}{t_{2}-t_{1}}\left\langle\xi, v^{0}\right\rangle-\frac{t-t_{1}}{t_{2}-t_{1}}\left\langle\xi, \tilde{f}\left(x\left(t_{2}\right)\right)\right\rangle .
$$

Since $\varphi$ is lsc, there exists a point $t \in\left[t_{1}, t_{2}\right]$ such that $\varphi(\hat{t})=\min \left\{\varphi(t) \mid t_{1} \leq t \leq t_{2}\right\}$. We have $\varphi(\hat{t}) \leq \varphi\left(t_{2}\right)<+\infty$. This property implies $\tilde{f}(x(\hat{t})) \in Y$.

Since $\left\langle\xi, \tilde{f}\left(x\left(t_{1}\right)\right)\right\rangle=\left\langle\xi, v_{\infty}^{0}\right\rangle$ (here we make use of $v^{0} \in \operatorname{int} C$ ), we have $0 \leq$ $t_{1}<\hat{t} \leq t_{2} \leq 1$. Set $\hat{x}=x(\hat{t})$ and

$$
\hat{y}=\lim _{k} \frac{1}{s_{k}}\left(\tilde{f}\left(\hat{x}+s_{k}\left(x^{*}-x\right)\right)-\tilde{f}(\hat{x})\right) \in \tilde{f}^{\prime}\left(\hat{x}, x^{*}-x\right)
$$


(the convergence is in $\tilde{Y}$ ). We may assume that $0<s_{k}<\hat{t}-t_{1}$ for all $k$. The minimal property of $\hat{t}$ gives $\varphi\left(\hat{t}-s_{k}\right) \geq \varphi(\hat{t})$, which can be written in the form

$$
\left\langle\xi, \frac{1}{s_{k}}\left(\tilde{f}\left(\hat{x}+s_{k}\left(x^{*}-x\right)\right)-\tilde{f}(\hat{x})\right)\right\rangle+\frac{1}{t_{2}-t_{1}}\left\langle\xi,\left(\tilde{f}\left(x\left(t_{2}\right)\right)-\lambda_{0} v^{0}\right)\right\rangle \geq 0 .
$$

We have $\hat{x} \in \operatorname{dom} \tilde{f}$. From the hypotheses, like in the proof of Theorem 5 , we see that $\hat{y} \in \tilde{f}^{\prime}\left(\hat{x}, x^{*}-x\right)$ can be chosen in such a way that the following alternative takes place:

$\mathbf{1}^{0} . \hat{y} \in-C$

$\mathbf{2}^{0} \cdot \hat{y}=\hat{v}_{\infty} \in-\operatorname{int} \tilde{C}$.

Assume that $\mathbf{1}^{0}$ holds. Then passing to a limit in (15) and applying $\langle\xi, \hat{y}\rangle \leq 0$ we get $\left\langle\xi, \tilde{f}\left(x\left(t_{2}\right)\right)-\lambda_{0} v^{0}\right\rangle \geq 0$, which is a contradiction to $\tilde{f}\left(x\left(t_{2}\right)\right)-\lambda_{0} v^{0} \in-\operatorname{int} C$.

Assume now that $\mathbf{2}^{0}$ holds. Then there exists an open cone $W \subset C$, such that $\hat{v} \in-W \subset-C$. From the definition of the convergence in $\tilde{Y}$, for sufficiently large $k$ we have $\left(1 / s_{k}\right)\left(\tilde{f}\left(\hat{x}+s_{k}\left(x^{*}-x\right)\right)-\tilde{f}(\hat{x})\right) \in-W \subset-C$. Therefore

$$
\left\langle\xi, \tilde{f}\left(x\left(t_{2}\right)\right)-\lambda_{0} v^{0}\right\rangle \geq-\left(t_{2}-t_{1}\right)\left\langle\xi, \frac{1}{s_{k}}\left(\tilde{f}\left(\hat{x}+s_{k}\left(x^{*}-x\right)\right)-\tilde{f}(\hat{x})\right)\right\rangle \geq 0 .
$$

Thus, we have shown that $\left\langle\xi, \tilde{f}\left(x\left(t_{2}\right)\right)-\lambda_{0} v^{0}\right\rangle \geq 0$ for all $\xi \in C^{\prime}$. This implies that $\tilde{f}\left(x\left(t_{2}\right)\right)-\lambda_{0} v^{0} \in C$. With the account of the choice of $\lambda_{0}$ we get $\lambda_{0} v^{0}-$ $\tilde{f}\left(x\left(t_{2}\right)\right) \in(-C) \cap \operatorname{int} C=\emptyset$, a contradiction.

C. Now we show that also the assumption $\tilde{f}\left(x\left(t_{2}\right)\right)=v_{\infty}^{0}$ leads to a contradiction. Indeed, if we assume that $\tilde{f}\left(x\left(t_{2}\right)\right)=v_{\infty}^{0}$, we face the situation when on the left end of the interval $\left[t_{2}, 1\right]$ we have $\tilde{f}\left(x\left(t_{2}\right)\right)=v_{\infty}^{0}$, while on the right end $\tilde{f}(x(1))=\tilde{f}(x) \in Y$. However in point $\mathbf{B}$ we have shown that this case does not occur.

Thus, for arbitrary $x \in K$, and for arbitrary $0 \leq t_{1}<t_{2} \leq 1$, we have shown that both $\tilde{f}\left(x\left(t_{1}\right)\right)$ and $\tilde{f}\left(x\left(t_{2}\right)\right)$ belong to $Y$, whence $\left[x^{*}, x\right] \subset K$. Moreover, we have seen that $\tilde{f}\left(x\left(t_{2}\right)\right) \subset \tilde{f}\left(x\left(t_{1}\right)\right)+C$. Therefore $\tilde{f} \in I A R^{+}\left(X, x^{*}\right)$. Now Theorem 6 gives also that $x^{*} \in \operatorname{ker} K$, and $x^{*}$ is an $a$-minimizer of the associated optimization problem (10).

When $Y=\boldsymbol{R}$ and $C=\boldsymbol{R}_{+}$the generalized vector VI (14) turns into the generalized scalar VI

$$
\tilde{f}^{\prime}\left(x, x^{*}-x\right) \cap\left(-\overline{\boldsymbol{R}}_{+}\right) \neq \emptyset, \quad x \in X .
$$

For this equation, we get the following corollary:

Corollary 3. Let $\tilde{f}: X \rightarrow \boldsymbol{R} \cup\{+\infty\}, x^{*} \in K=\operatorname{dom} \tilde{f}$, and $\tilde{f} \in R L S C\left(X, x^{*}\right)$. Suppose that $x^{*} \in K$ is a solution of the generalized scalar VI (16). Then $\tilde{f} \in$ $I_{A} R^{+}\left(X, x^{*}\right)$. Further $x^{*} \in \operatorname{ker} K$, and $x^{*}$ is an a-minimizer of the optimization problem $\min \tilde{f}, x \in X$. 
Remark 4. In [4] we proved Corollary 3 under the additional assumption that $\operatorname{dom} \tilde{f}$ is radially closed along the rays starting at $x^{*}$. This means, that for all $u \in X$ the set $\left\{t \in \boldsymbol{R}_{+} \mid x^{*}+t u \in \operatorname{dom} \tilde{f}\right\}$ is closed. Here we see that this hypothesis is superfluous.

\section{QuASICONVEX FUnCtions}

We have seen that each solution $x^{*}$ of the extended VI $(7)$ is an $a$-minimizer. The reverse in general is not true. For instance, in Example 7 the points $(0,1)$ and $(1,0)$ are $a$-minimizers, but not solutions of $(7)$. Here we show, that the reverse is still true, when the function $f: K \rightarrow Y$ is quasiconvex and $Y=\boldsymbol{R}^{m}$.

We say that the function $f: K \rightarrow Y$ is quasiconvex (with respect to the cone $C$ ) if the set $K$ is convex and for each $y \in Y$ the set $\{x \in K \mid f(x) \in y-C\}$ is convex.

Theorem 9. Let $Y=\boldsymbol{R}^{m}$ and $f: K \rightarrow Y$ be quasiconvex. Suppose that $x^{*}$ is an a-minimizer of (4). Then $x^{*}$ is a solution of the extended VI (7).

Proof. Since $K$ is convex, the kernel of $K$ coincides with $K$ and in particular $x^{*} \in \operatorname{ker} K$. Let $x \in K, x(t)=(1-t) x^{*}+t x$ and $0 \leq t_{1}<t_{2} \leq 1$. Since $x^{*}$ is an $a$-minimizer we have $f\left(x^{*}\right)=f(x(0)) \in f\left(x\left(t_{2}\right)\right)-C$. Therefore both $x(0)$ and $x\left(t_{2}\right)$ belong to the set $\left\{x \in K \mid f(x) \in f\left(x\left(t_{2}\right)\right)-C\right\}$. Since $f$ is quasiconvex, $x\left(t_{1}\right) \in\left[x(0), x\left(t_{2}\right)\right]$ belongs to this set, in other words $f\left(x\left(t_{1}\right)\right) \in f\left(x\left(t_{2}\right)\right)-C$. Therefore $f \in I A R^{+}\left(K, x^{*}\right)$. Now Theorem 4 gives that $x^{*}$ is a solution of the extended vector VI (7).

\section{Final Remarks}

Most of the proofs in this paper were based on the following scheme. Instead of considering directly the extended vector VI (7), we deal with the system of scalar VI

$$
\phi^{\prime}\left(x, x^{*}-x\right) \leq 0, \quad x \in K, \quad \text { for all } \phi \in \Phi\left(\Xi, x^{*}\right),
$$

where $\Phi\left(\Xi, x^{*}\right)$ is the set of functions $\phi: K \rightarrow \boldsymbol{R}$ defined by $\phi(x)=\left\langle\xi, f(x)-f\left(x^{*}\right)\right\rangle$, $\xi \in \Xi:=C^{\prime}$. Then $f \in I_{A} R^{+}\left(K, x^{*}\right)$ if and only if $\phi \in I A R\left(K, x^{*}\right)$ for all $\phi \in \Phi\left(\Xi, x^{*}\right)$. The point $x^{*}$ is an $a$-minimizer of $f$ if and only if $x^{*}$ is a global minimizer of $\phi$ for all $\phi \in \Phi\left(\Xi, x^{*}\right)$.

This situation can be generalized. Let $\Xi$ be a set of functions $\xi: Y \rightarrow \boldsymbol{R}$. For $x^{*} \in \operatorname{ker} f$ define the set $\Phi\left(\Xi, x^{*}\right)$ of all functions $\phi: K \rightarrow \boldsymbol{R}$, such that $\phi(x)=\xi\left(f(x)-f\left(x^{*}\right)\right)$ for some $\xi \in \Xi$. Now we say that $f$ is increasing-along-rays (IAR) with respect to $\Xi(\Xi-I A R)$ starting at $x^{*}$ in $K$, if $\phi \in I A R\left(K, x^{*}\right)$ for all $\phi \in \Phi\left(\Xi, x^{*}\right)$. We say that $x^{*}$ is a minimizer of $f$ with respect to $\Xi$ if $x^{*}$ is a minimizer of $\phi$ for all $\phi \in \Phi\left(\Xi, x^{*}\right)$. Now we can consider the system of scalar VI (17). One can try for (17) to establish relations between the properties: $x^{*}$ is a solution of (17) in the sense that $x^{*}$ satisfies each equation of the system; $f$ is 
IAR with respect to $\Xi$ at $x^{*}$ in $K ; x^{*}$ is a minimizer of $f$ with respect to $\Xi$. One expects results similar to those from the present paper.

The attempt to define a class $\Xi$ such that a result analogous to that of Section 6 holds, may however produce some obstacles. At first glance one thinks to define quasiconvexity with respect to $\Xi$ and $x^{*}$ to be quasiconvexity of all the functions $\phi \in \Phi\left(\Xi, x^{*}\right)$ as real-valued functions. However one sees easily, that if $\Xi$ contains nonlinear functions the generalization of Theorem 9 is not so straightforward.

The system of scalar VI (17) with arbitrary set $\Xi$ can be used to stress new features of the vector optimization problem (4). For instance, we have seen that the solutions of the considered vector VI are $a$-minimizers. If the vector optimization problem (4) possesses $a$-minimizers we will certainly wish to distinguish them, since they represent a rather nice property. Roughly speaking, the $a$-minimizer is "better" than any other point, while the $w$-minimizer is "not worse" than any other point. Unfortunately, often $a$-minimizers do not exist and when solving the vector optimization problem we will be satisfied to find some point of efficiency of other type. Besides $w$-minimizers and $e$-minimizers, in the literature numerous types of points of efficiently are known and for their description often scalarization technique is applied, for a survey see [23]. If

$$
\min \xi_{0}\left(f(x)-f\left(x^{*}\right)\right), \quad x \in K,
$$

is a scalarization associated with the point $x^{*}$, then we may consider (17) in which $\Xi=\left\{\xi_{0}\right\}$ is a singleton. Now (17) transforms into a single scalar variational inequality. If we consider this single variational inequality under lsc assumptions, then $x^{*}$ is its solution if and only if $x^{*}$ is a point of efficiency of the considered type for the vector optimization problem.

For instance, suppose we deal with the case $Y=\boldsymbol{R}^{m}$ endowed with the norm $\|\cdot\|$ (recall that any two norms in $\boldsymbol{R}^{m}$ are equivalent) and we wish to study $w$ minimizers. Naturally, we would not be satisfied from the approach in Section 2, since, as Example 2 showed, Theorem 2 cannot be reverted. Instead, we can apply as in [3] a scalarization (18) in which

$$
\xi_{0}(y)=\max \left\{\langle\xi, y\rangle \mid \xi \in C^{\prime},\|\xi\|=1\right\} .
$$

In fact $\xi_{0}(y)$ represents the Hiriart-Urruty oriented distance [10] from the point $y$ to the cone $-C$. Pay attention that $\xi_{0}$ is a nonlinear function. The corresponding VI appears in [5], where it is illustrated that such a particular VI poses new problems in relation to the vector optimization problem. For instance, by definition, we see that the notion of a $w$-minimizer of $f$ or the property of $f$ to be quasiconvex (with respect to $C$ ) is norm-independent, while the property of $f$ to be IAR with respect to $\Xi$ at $x^{*}$, and the property of $f$ to be quasiconvex with respect to $\Xi$ and $x^{*}$ happen to be norm-dependent (here we speak for the case when $\Xi=\left\{\xi_{0}\right\}$ and $\xi_{0}$ is given by (19)). This circumstance rises the question, under which conditions, for any given $w$-minimizer $x^{*}$ there exists a norm in $Y$ such that $f$ is IAR with respect to $\Xi$ at $x^{*}$. We can also list some other related problems: if $f$ is quasiconvex (with respect to $C$ ), then the question is when one can choose the norm in $Y$ in such 
a way that $f$ is also quasiconvex with respect to $\Xi$. Moreover, since the function $\xi_{0}$ is not linear, the question is, under the assumption that $f$ is quasiconvex with respect to $\Xi$ and that $x^{*}$ is a minimizer with respect to $\Xi$ of $f$, whether there exists a norm in $Y$, such that $f$ is IAR with respect to $\Xi$ at $x^{*}$.

\section{REFERENCES}

[1] C. Baiocchi and A. Capelo, Variational and Quasivariational Inequalities, Applications to Free-Boundary Problems. John Wiley \& Sons, New York (1984).

[2] G.P. Crespi, I. Ginchev and M. Rocca, Minty vector variational inequality, efficiency and proper efficiency. Vietnam J. Math. 32 (2004) 95-107.

[3] G.P. Crespi, I. Ginchev and M. Rocca, Variational inequalities in vector optimization, in Variational Analysis and Applications, Series: Nonconvex Optimization and its Applications 79, edited by F. Giannessi and A. Maugeri. Springer, New York (2005), Part II, $259-278$.

[4] G.P. Crespi, I. Ginchev and M. Rocca, Minty variational inequalities, increase along rays property and optimization. J. Optim. Theory Appl. 123 (2004) 479-496.

[5] G.P. Crespi, I. Ginchev and M. Rocca, Increase-along-rays property for vector functions, Preprint 2004/24, Universitá dell'Insubria, Facoltá di Economia, Varese, 2004, (http://eco.uninsubria.it/dipeco/Quaderni/files/QF2004_24.pdf).

[6] F. Giannessi, Theorems of alternative, quadratic programs and complementarity problems, in Variational Inequalities and Complementarity Problems, edited by R.W. Cottle, F. Giannessi and J.-L. Lions, John Wiley \& Sons, New York (1980) 151-186.

[7] F. Giannessi, On Minty variational principle, in New Trends in Mathematical Programming, edited by F. Giannessi, S. Komlósi, and T. Rapcsák, Kluwer, Dordrecht (1998) 93-99.

[8] I. Ginchev, Higher order optimality conditions in nonsmooth vector optimization, in Generalized Convexity, Generalized Monotonicity, Optimality Conditions and Duality in Scalar and Vector Optimization, edited by A. Cambini, B.K. Dass and L. Martein. J. Stat. Manag. Syst. 5 (2002) 321-339.

[9] I. Ginchev, A. Guerraggio and M. Rocca, First-order conditions for $C^{0,1}$ constrained vector optimization, in Variational Analysis and Applications, Series: Nonconvex Optimization and its Applications 79, edited by F. Giannessi and A. Maugeri. Springer, New York (2005), Part II, 437-450.

[10] J.-B. Hiriart-Urruty, New concepts in nondifferentiable programming, Analyse non convexe. Bull. Soc. Math. France 60 (1979) 57-85.

[11] D. Kinderlehrer and G. Stampacchia, An Introduction to Variational Inequalities and Their Applications, Academic Press, New York (1980).

[12] A.N. Kolmogorov and S.V. Fomin, Elements of the theory of functions and of functional analysis, Nauka, Moscow (1972) (In Russian).

[13] S. Komlósi, On the Stampacchia and Minty variational inequalities, in Generalized Convexity and Optimization for Economic and Financial Decisions, Proc. Verona, Italy, May 28-29, 1998, edited by G. Giorgi and F. Rossi, Pitagora Editrice, Bologna (1999) 231-260.

[14] D.T. Luc, Theory of Vector Optimization. Springer-Verlag, Berlin (1989).

[15] G. Mastroeni, Some remarks on the role of generalized convexity in the theory of variational inequalities, in Generalized Convexity and Optimization for Economic and Financial Decisions. Proc. Verona, Italy, May 28-29, 1998, edited by G. Giorgi and F. Rossi, Pitagora Editrice, Bologna (1999) 271-281.

[16] G.J. Minty, On the generalization of a direct method of the calculus of variations. Bull. Amer. Math. Soc. 73 (1967) 314-321.

[17] B. Mordukhovich, Stability theory for parametric generalized equations and variational inequalities via nonsmooth analysis. Trans. Amer. Math. Soc. 343 (1994) 609-657.

[18] A.M. Rubinov, Abstract Convexity and Global Optimization, Kluwer, Dordrecht (2000). 
[19] H.H. Schaefer, Topological Vector Spaces. The MacMillan Company, New York, London, (1966).

[20] G. Stampacchia, Formes bilinéaires coercitives sur les ensembles convexes. C. R. Acad. Sci. Paris (Groupe 1) 258 (1960) 4413-4416.

[21] P.T. Thach and M. Kojima, A generalized convexity and variational inequality for quasiconvex minimization. SIAM J. Optim. 6 (1996) 212-226.

[22] X.Q. Yang, Generalized convex functions and vector variational inequalities. J. Optim. Theory Appl. 79 (1993) 563-580.

[23] A. Zaffaroni, Degrees of efficiency and degrees of minimality. SIAM J. Control Optim. 42 (2003) 1071-1086.

To access this journal online: www.edpsciences.org 